\title{
The Evolution of Luminous Compact Blue Galaxies: Disks or Spheroids?
}

\author{
D. J. Pisano ${ }^{1}$, K. Rabidoux ${ }^{1}$, C. A. Garland ${ }^{2}$, R. Guzmán ${ }^{3}$, \\ F. J. Castander ${ }^{4}$, and J. Pérez-Gallego ${ }^{3}$ \\ ${ }^{1}$ West Virginia University Dept. of Physics, P.O. Box 6315, Morgantown, WV 26506, USA \\ email: djpisano@mail.wvu.edu, krabidou@mix.wvu.edu \\ ${ }^{2}$ Natural Sciences Department, Castleton State College, Castleton, VT 05735, USA \\ email: catherine.garland@castleton.edu \\ ${ }^{3}$ Dept. of Astronomy, University of Florida, 211 Bryant Space Science Center, P.O. Box \\ 112055, Gainesville, FL 32611, USA \\ email: guzman@astro.ufl.edu, jgallego@astro.ufl.edu \\ ${ }^{4}$ Institut de Ciéncies de l'Espai (ICE/CSIC), Campus UAB, 08193 Bellaterra, Barcelona, Spain \\ email : fjc@ieec.fcr.es
}

\begin{abstract}
Luminous compact blue galaxies (LCBGs) are a diverse class of galaxies characterized by high luminosity, blue color, and high surface brightness that sit at the critical juncture of galaxies evolving from the blue to the red sequence. As part of our multi-wavelength survey of local LCBGs, we have been studying the HI content of these galaxies using both singledish telescopes and interferometers. Our goals are to determine if single-dish HI observations represent a true measure of the dynamical mass of LCBGs and to look for signatures of recent interactions that may be triggering star formation in LCBGs. Our data show that while some LCBGs are undergoing interactions, many appear isolated. While all LCBGs contain HI and show signatures of rotation, the population does not lie on the Tully-Fisher relation nor can it evolve onto it. Furthermore, the HI maps of many LCBGs show signatures of dynamically hot components, suggesting that we are seeing the formation of a thick disk or spheroid in at least some LCBGs. There is good agreement between the HI and $\mathrm{H} \alpha$ kinematics for LCBGs, and both are similar in appearance to the $\mathrm{H} \alpha$ kinematics of high redshift star-forming galaxies. Our combined data suggest that star formation in LCBGs is primarily quenched by virial heating, consistent with model predictions.
\end{abstract}

Keywords. galaxies: formation - galaxies: evolution - galaxies: ISM - galaxies: kinematics and dynamics - galaxies: interactions - galaxies: starburst

\section{Introduction}

When the universe was 4.6 Gyr old, the galaxy population was dominated by blue, star-forming galaxies. Up to $40 \%$ of these galaxies were luminous compact blue galaxies (LCBGs) which contribute significantly to the global star formation rate density at that time (Guzman et al. 1997). Today, the population of galaxies is roughly evenly divided between a red and a blue population and the star formation rate density has dropped by an order of magnitude. Similarly, LCBGs are an order of magnitude less common (Werk et al. 2004) and contribute negligibly to the global star formation rate (Guzman et al. 1997). LCBGs are a diverse class of galaxies characterized by their high luminosities $\left(\mathrm{M}_{B} \leqslant-18.5 \mathrm{mag}\right)$, compact sizes $\left(\mathrm{SBe}(\mathrm{B}) \leqslant 21 \mathrm{mag} \operatorname{arcsec}^{-2}\right.$, equivalent to $\left.\mathrm{r}_{e f f} \leqslant 4 \mathrm{kpc}\right)$, and blue colors $(B-V \leqslant 0.6 \mathrm{mag})$; they have the highest star formation rate per unit mass for high mass galaxies (Gil de Paz et al. 2000). Typical stellar masses of LCBGs are $\sim 5 \times 10^{10} M_{\odot}$, (Guzmán et al. 2003) placing them near the maximal stellar mass of 
the blue sequence (Kauffmann et al. 2003). Above this mass limit, all galaxies are red so some process must quench the star formation in galaxies as they grow. There have been numerous theories as to what quenching mechanisms operate in galaxies on the blue sequence. These include the shock heating of gas to the virial temperature (Cattaneo et al. 2006), or heating by starbursts by supernovae- or AGN-driven winds or some combination of multiple processes (Hopkins et al. 2006, and references therein). Since LCBGs reside at the high mass end of the blue sequence, they are poised to have their star formation quenched in the near future and, therefore, represent an ideal population to study viable quenching mechanisms that could also be responsible for the emergence of a red sequence in the past 8 Gyr.

We are conducting a multi-wavelength survey, spanning the ultraviolet through the radio, of the rare, local LCBGs to constrain the viable mechanisms for quenching star formation in blue galaxies and the future evolutionary paths of LCBGs. Therefore, we have selected our LCBGs from the Sloan Digital Sky Survey (SDSS) within D $\leqslant 200$ Mpc to have the same properties, listed above, as LCBGs at high redshift. This yields a total of 2359 LCBGs out of over 800,000 galaxies in the SDSS DR4. Of these, we have collected single-dish HI observations of 163 LCBGs. The distribution of properties for all LCBGs are shown in Figure 1.

\section{Results}

Single-dish HI observations permit a direct measure of the amount of fuel available for star formation, $M_{H I}$, and, by using the linewidth and an estimate of a galaxy's extent, the dynamical mass, $M_{d y n}$. When combined with a measure of the star formation rate, such as the non-thermal radio continuum emission or emission from dust in the far-infrared, this provides an estimate of the duration of the current starburst: $\tau=M_{H I} / S F R$.

We have used original observations and archival data from Arecibo, the Green Bank Telescope, Parkes, Nançay, and the old Green Bank 140-foot and 300-foot telescopes
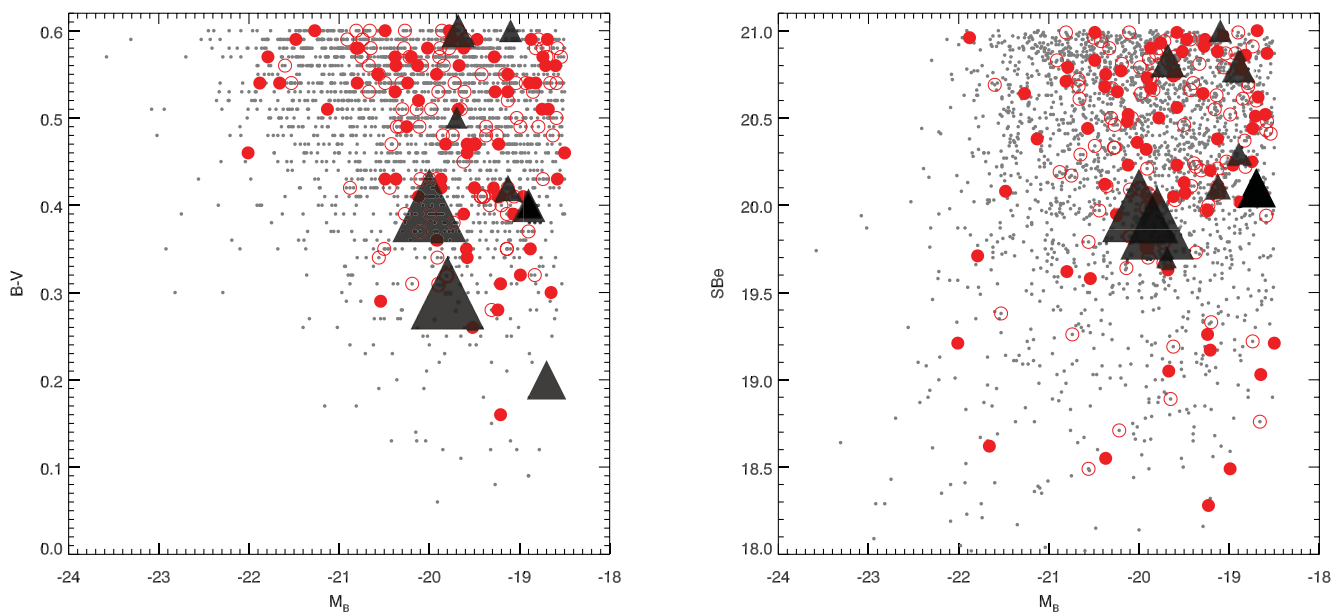

Figure 1. Left: $B-V$ vs. $\mathrm{M}_{B}$ for all LCBGs selected from the SDSS DR4 (grey dots) and all LCBGs with single-dish HI data (circles). The filled circles are those LCBGs with companions within the beam, the open circles are isolated. The triangles represent those LCBGs with GMRT or VLA data with the size of the triangle inversely proportional to $\mathrm{V}_{\text {rot }} / \sigma$. Right: Same as the left but for $\mathrm{SBe}(\mathrm{B})$ vs. $\mathrm{M}_{B}$. 
(Springob et al. 2005, Giovanelli et al. 2005) in concert with radio continuum fluxes from NVSS and far-infrared fluxes from IRAS to measure $M_{H I}, M_{d y n}$, and $\tau$ for 163 LCBGs. Of the 63 galaxies we observed ourselves, we detected $94 \%$ of them at the $5 \sigma$ level of $2.5 \times 10^{8} M_{\odot}$. LCBGs have a wide range of properties with an average $M_{H I} \sim 10^{9.7} M_{\odot}$ and an average $M_{d y n} \sim 10^{10.6} M_{\odot} ; 80 \% \tau \leqslant 3 \mathrm{Gyr}$ (Garland et al. 2004, Pisano et al. 2011 , in preparation). All LCBGs have $M_{d y n} \leqslant 10^{12} M_{\odot}$, below the maximal halo mass predicted by Cattaneo et al. (2006), suggesting that virial heating is a viable quenching mechanism. Finally, there is no significant difference between the HI properties of LCBGs with and without nearby companions; about 50\% of LCBGs have close opticallybright companions. These properties suggest that LCBGs may evolve into low-mass spiral galaxies or high-mass dwarf ellipticals or, possibly, bulges. Given the wide range of properties of LCBGs, the specific evolutionary path of a galaxy depends critically on its exact properties, such that each LCBG will follow a different evolutionary path.

There is, however, a caveat to this relatively simple evolutionary picture. LCBGs lie well above and below the Tully-Fisher relation, so many cannot even evolve onto it. This may be due to nearby companions confusing measurements of the integrated HI linewidth or from significant deviations from pure circular rotation in the LCBGs due to recent interactions. In order to quantify these effects, we have mapped a total of 18 LCBGs with the VLA or the GMRT. Initial analysis for three interacting LCBGs was presented in Garland et al. (2007), and we present the results for eight additional LCBGs here.

As can be seen from Figure 2, while some LCBGs have regular rotation and relatively small velocity dispersions others have a far more complex velocity structure, even if they lack a close companion. Our high resolution GMRT observations reveal that the singledish linewidths are about $15 \%$ larger than what would be inferred from the interferometer data. This overestimate of the rotation velocity is not large enough, however, to explain the dispersion seen around the Tully-Fisher relation.

Our observations also show that LCBGs tend to have high velocity dispersions, $\sim 20$ $40 \mathrm{~km} \mathrm{~s}^{-1}$, across much of the galactic disk. For those LCBGs with large rotation velocities, this leads to $V_{\text {rot }} / \sigma \sim 5-7$ (seen in the top row of Figure 2), however many LCBGs have much smaller values of $V_{\text {rot }} / \sigma$ (seen in the bottom row of Figure 2 ) of $\lesssim 2$. Similar features are seen in optical velocity fields of star-forming high-redshift galaxies (Shapiro et al. 2008, Gonçalves et al. 2010, and these proceedings). Such low $V_{\text {rot }} / \sigma$ values indicate the presence of a dynamically hot component in a number of LCBGs, perhaps indicating the formation of a thick disk or a bulge after a recent minor merger. Our HI velocity fields agree with the $\mathrm{H} \alpha$ velocity fields observed by Pérez-Gallego et al. (2011), suggesting that the dynamics of the ionized gas are not severely affected by galactic outflows.

\section{Conclusions}

The signature of ongoing spheroid formation in some LCBGs is consistent with the idea that star formation in these galaxies is being quenched via virial heating, but this is not a unique explanation. Figure 1 shows that those LCBGs with the smallest values of $V_{\text {rot }} / \sigma$ are the most compact, bluest, and highest luminosity systems. This could also indicate that quenching from heating due to the intense central starburst or its associated supernovae is a possibility. This is supported by the results of optical spectroscopy by Pérez-Gallego et al. (2011) who found that while only 5\% of LCBGs have an AGN, $27 \%$ have signatures of supernovae- driven winds. The remaining LCBGs could then be quenched via virial heating. In the future, we will be expanding our HI mapping to study 

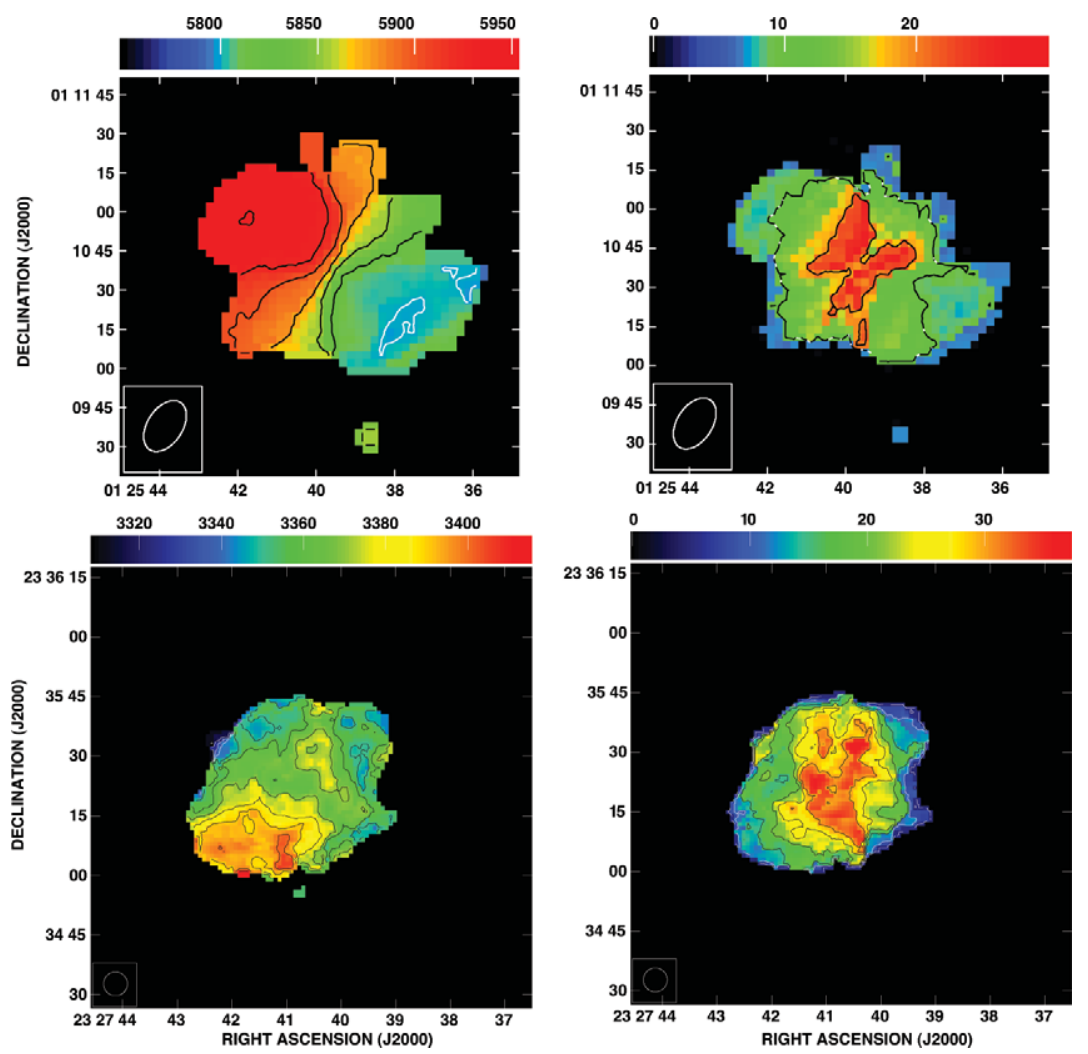

Figure 2. A montage of velocity fields (left column) and maps of velocity dispersions (right column) for SDSS $0125+0110$ (top row) and Mrk 325 (bottom row). Velocity field contours are every $25 \mathrm{~km} \mathrm{~s}^{-1}$ for SDSS $0125+0110$ and every $10 \mathrm{~km} \mathrm{~s}^{-1}$ for Mrk 325. Contours on the velocity dispersion maps are every $10 \mathrm{~km} \mathrm{~s}^{-1}$ and every $5 \mathrm{~km} \mathrm{~s}^{-1}$ for the two galaxies, respectively. The beam size is shown in the lower left corner of each panel.

additional LCBGs with a wider range of properties and we will use multi-wavelength data to search for signatures of active quenching in LCBGs.

\section{References}

Cattaneo, A., Dekel, A., Devriendt, J., Guiderdoni, B., \& Blaizot, J. 2006, MNRAS, 370, 1651

Garland, C. A., Pisano, D. J., Williams, J. P., Guzmán, R., \& Castander, F. J. 2004, ApJ, 615, 689

Garland, C. A., Pisano, D. J., Williams, J. P., Guzmán, R., Castander, F. J., \& Sage, L. J. 2007, ApJ, 671, 310

Gil de Paz, A., Aragón-Salamanca, A., Gallego, J., Alonso-Herrero, A., Zamorano, J., \& Kauffmann, G. 2000, MNRAS, 316, 357

Giovanelli, R., et al. 2005, AJ, 130, 2598

Gonçalves, T. S., et al. 2010, ApJ, 724, 1373

Guzman, R., Gallego, J., Koo, D. C., Phillips, A. C., Lowenthal, J. D., Faber, S. M., Illingworth, G. D., \& Vogt, N. P. 1997, ApJ, 489, 559

Guzmán, R., Östlin, G., Kunth, D., Bershady, M. A., Koo, D. C., \& Pahre, M. A. 2003, ApJ, $586, \mathrm{~L} 45$ 
Hopkins, P. F., Hernquist, L., Cox, T. J., Di Matteo, T., Robertson, B., \& Springel, V. 2006, ApJS, 163, 1

Kauffmann, G., et al. 2003, MNRAS, 341, 54

Pérez-Gallego, J., et al., 2011, MNRAS, submitted

Shapiro, K. L., et al. 2008, ApJ, 682, 231

Springob, C. M., Haynes, M. P., Giovanelli, R., \& Kent, B. R. 2005, ApJS, 160, 149

Werk, J. K., Jangren, A., \& Salzer, J. J. 2004, ApJ, 617, 1004 